\title{
Mid-infrared Waveguide Evanescent Field Chemical Sensor for Liquids
}

\author{
Yu-Chi Chang ${ }^{1}$, Vincent Paeder ${ }^{1}$, Lubos Hvozdara ${ }^{1}$, Philip Wägli ${ }^{2}$, Alexandra Homsy ${ }^{2}$ \\ Jean-Michel Hartmann ${ }^{3}$, Hans Peter Herzig ${ }^{1}$ \\ ${ }^{I}$ Optics and Photonics Technology Laboratory, Ecole Polytechnique Fédérale de Lausanne, Brequet 2, 2000 Neuchâtel, Switzerland \\ ${ }^{2}$ Sensors, Actuators and Microsystems Laboratory,Ecole Polytechnique Fédérale de Lausanne, Jaquet-Droz 1, 2002 Neuchâtel, Switzerland \\ ${ }^{3}$ CEA-LETI, Minatec, 17 Rue des Martyrs, 38054 Grenoble, France
}

Based on a mid-infrared waveguide fabricated from Germanium on Silicon substrates, we present a concept of chemical sensors in aqueous solutions. The sensing scheme is utilizing the evanescent wave in a single-mode waveguide, and the absorption spectrum of chemicals dissolved in liquids. The waveguide is coupled to a singlemode mid-infrared quantum cascade laser [1], and an infrared detector. The wavelength of 5.81 microns is chosen to assure the specifity and sensitivity of the pilot application [2] for the analysis of cocaine analyte, in a water based matrix (saliva). A micro-fluidic system will be used for sample preparations.

The left part of figure 1 shows the sensing scheme of the proposed device. The analyte is detected in the evanescent field when the laser wavelength is chosen to its specific absorption peaks. In the right side of figure 1, SEM photos show Ge waveguides fabricated on $\mathrm{Si}$ substrates and the cross-section view. A typical photolithography process is used with reactive ion etching of CF4 chemistry.
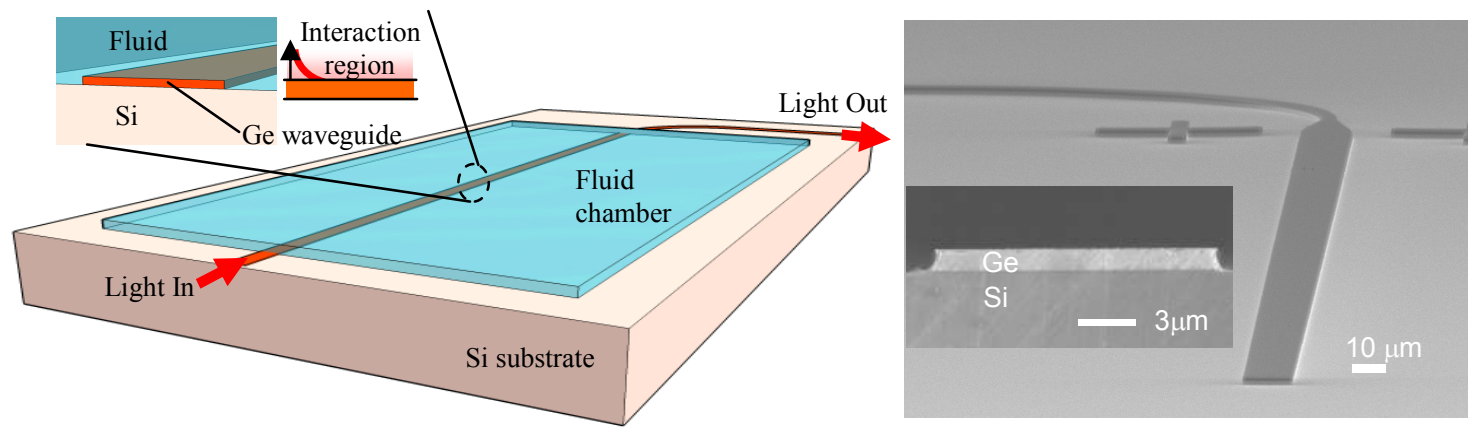

Fig. 1 The sensing scheme of a evanescent field chemical sensor, and SEM photos of a Ge waveguide on Si substrates

The waveguide facets are designed to have a better couple efficiency with thickness of 1.1 microns and width of 15 microns. It is then tapered narrower to have a single-mode waveguide. A waveguide bend is designed to avoid the stray light going to the detector.

A quantum cascade laser has been coupled into the waveguide with a butt-coupling configuration. Fig.2 shows that the gap between the laser and the waveguide is not visible. The light passing through the waveguide is then collected by two off-axis parabolic mirrors and going into a Mercury-Cadmium-Telluride (MCT) infrared detector.

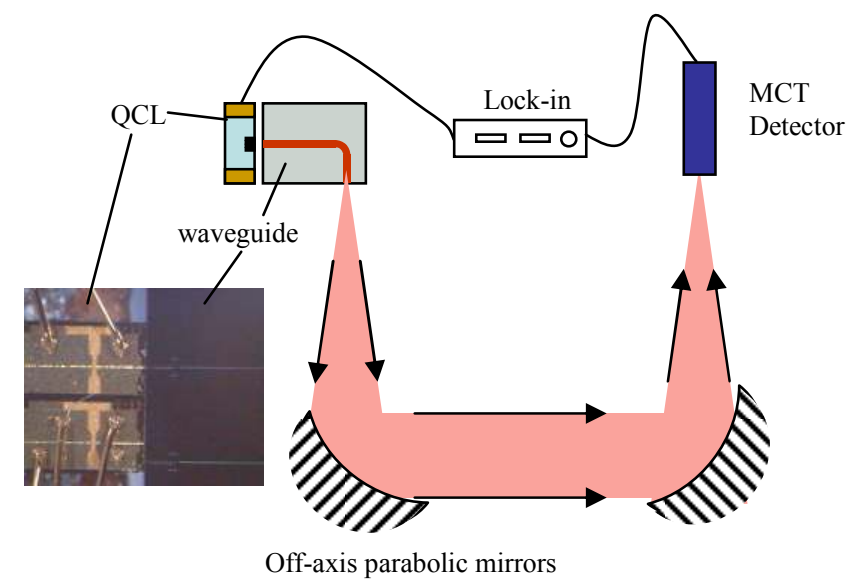

Fig. 2 The setup for optical characterization with a butt-coupling of the waveguide and the quantum cascade laser

\section{References}

[1] J. Faist, F. Capasso, D.L. Sivco, C. Sirtori, A.L. Hutchinson, and A.Y.Cho, “Quantum Cascade Laser,” Science 264, 553 (1994)

[2] http://www.nano-tera.ch/projects/80.php 